\title{
Dynamical Symmetry Restoration for a Higher-Derivative Four-Fermion Model in an External Electromagnetic Field
}

\author{
E. Elizalde ${ }^{1,2, *}$ S. P. Gavrilov ${ }^{3,4, \dagger}$ S. D. Odintsov ${ }^{1,4, \ddagger}$ and Yu. I. Shil'nov ${ }^{1,5, \S}$ \\ ${ }^{1}$ Consejo Superior de Investigaciones Científicas, \\ IEEC, Edifici Nexus-204, Gran Capità 2-4, 08034, Barcelona, Spain \\ ${ }^{2}$ Department ECM, Faculty of Physics, University of Barcelona, \\ Diagonal 647, 08028, Barcelona, Spain \\ ${ }^{3}$ Departamento de Física, CCET, Universidade Federal de Sergipe, \\ 490000-000 Aracaju, SE, Brasil \\ ${ }^{4}$ Department of Mathematics and Physics, \\ Tomsk Pedagogical University, 634041, Tomsk, Russia \\ ${ }^{5}$ Department of Theoretical Physics, Faculty of Physics, \\ Kharkov State University, Svobody Sq. 4, 310077, Kharkov, Ukraine
}

Received 31 March, 2000

\begin{abstract}
A four-fermion model with additional higher-derivative terms is investigated in an external electromagnetic field. The effective potential in the leading order of large- $N$ expansion is calculated in external constant magnetic and electric fields. It is shown that, in contrast to the former results concerning the universal character of "magnetic catalysis" in dynamical symmetry breaking, in the present higher-derivative model the magnetic field restores chiral symmetry broken initially on the tree level. Numerical results describing a second-order phase transition that accompanies the symmetry restoration at the quantum level are presented.
\end{abstract}

\section{Introduction}

The dynamical symmetry breaking (DSB) issue has attracted a lot of attention since the appearence of the seminal paper by Nambu and Jona-Lasinio [1]. It is, in fact, the most economical way to realize the Higgs mechanism. It has been applied to the study of different models of modern quantum field theory [3], especially four-fermion ones $[1,2]$, where it is viewed as a lowenergy effective action of strong interactions physics [4].

Chiral symmetry (CS) breaking and dynamical fermion mass generation in four-fermion models have been investigated in the presence of external fields: electromagnetic [5]-[11], gravitational [12] - [14], and their combination [14], [15]. It has been observed that both a positive spacetime curvature and an external electric field try to restore chiral symmetry while a magnetic field and negative curvature always break it. Therefore external fields have been shown to induce some new

\footnotetext{
*E-mail: eli@zeta.ecm.ub.es, elizalde@io.ieec.fcr.es

†E-mail: gavrilov@ufs.br

$\ddagger$ E-mail: odintsov@tspi.tomsk.su

$\S$ E-mail: shil@kink.univer.kharkov.ua, visit2@ieec.fcr.es
}

phase transitions and enrich the model phase diagram essentially.

Higher-derivative extensions of the NJL model have been discussed recently owing to different reasons. First, a model with higher-derivative terms in the interaction vertex was proposed that exhibits an intersting equivalence with the symplified theory of electroweak interactions [16]. These terms of the low-energy effective action were shown to be essential and even unavoidable in the strong coupling regime, where a nontrivial phase diagram with a policritical point was shown to exist [17]. On the other hand, some regularization schemes based on the introduction of additional higherderivative terms into the initial kinetic one were discussed [18]. Furthemore, gravity effects upon the DSB for the former variant of high-derivative NJL model were investigated as well [19].

In the present paper we study the DSB in the fourfermion model with a higher-derivative kinetic term [18] 
in an external electromagnetic field. It is of interest to check up if the "universal rules" concerning magnetic catalysis of DSB and chiral symmetry restoration under the influence of electric fields work for this more complicated model.

\section{Effective potential of the higher-derivative model}

We are going to investigate a higher-derivative generalization of the NJL-like model in an external constant electromagnetic field with the following action:

$$
S=\int d^{4} x\left\{\bar{\psi}\left[\gamma^{\mu} i D_{\mu}+\xi\left(\gamma^{\mu} i D_{\mu}\right)^{2}\right] \psi+\frac{\lambda}{2 N}\left[(\bar{\psi} \psi)^{2}+\left(\bar{\psi} i \gamma_{5} \psi\right)^{2}\right]\right\},
$$

where the covariant derivative $D_{\mu}$ includes the electromagnetic potential $A_{\mu}$ :

$$
D_{\mu}=\partial_{\mu}-i e A_{\mu} .
$$

It should be noted that in our model CS is already broken on the tree level when $\xi \neq 0$ in contrast to the original NJL case. By introducing the auxiliary fields

$$
\sigma=-\frac{\lambda}{N}(\bar{\psi} \psi), \quad \pi=-\frac{\lambda}{N} \bar{\psi} i \gamma_{5} \psi,
$$

we can rewrite the action as

$$
S=\int d^{4} x\left\{\bar{\psi}\left[\gamma^{\mu} i D_{\mu}+\xi\left(\gamma^{\mu} i D_{\mu}\right)^{2}-\left(\sigma+i \pi \gamma_{5}\right)\right] \psi-\frac{N}{2 \lambda}\left(\sigma^{2}+\pi^{2}\right)\right\} .
$$

Then, the effective action in the large- $N$ expansion is given by

$$
\frac{1}{N} \Gamma_{e f f}(\sigma, \pi)=-\int d^{4} x \frac{\sigma^{2}+\pi^{2}}{2 \lambda}-i \ln \operatorname{det}\left\{i \gamma^{\mu}(x) D_{\mu}+\xi\left(\gamma^{\mu} i D_{\mu}\right)^{2}-\left(\sigma+i \gamma_{5} \pi\right)\right\} .
$$

Here we can put $\pi=0$, because the final expression will depend on the combination $\sigma^{2}+\pi^{2}$ only.

Defining the effective potential (EP) as $V_{e f f}=-\Gamma_{e f f} / N \int d^{4} x$, for constant configurations of $\sigma$ and $\pi$ we get

$$
V_{e f f}=\frac{\sigma^{2}}{2 \lambda}+i \operatorname{Sp} \ln \left\langle x\left|\left[\gamma^{\mu} i D_{\mu}+\xi\left(\gamma^{\mu} i D_{\mu}\right)^{2}-\sigma\right]\right| x\right\rangle .
$$

By means of the Green function (GF) which obeys the equation

$$
\left(i \gamma^{\mu} D_{\mu}+\xi\left(\gamma^{\mu} i D_{\mu}\right)^{2}-\sigma\right)_{x} G\left(x, x^{\prime}, \sigma\right)=\delta\left(x-x^{\prime}\right),
$$

we obtain the following formula

$$
V_{e f f}^{\prime}(\sigma)=\frac{\sigma}{\lambda}-i \operatorname{Sp} G(x, x, \sigma)
$$

To find out the GF $G\left(x, x^{\prime}, \sigma\right)$ it is convenient to represent the higher-derivative operator in the form

$$
i \gamma^{\mu} D_{\mu}+\xi\left(\gamma^{\mu} i D_{\mu}\right)^{2}-\sigma=\xi\left(i \gamma^{\mu} D_{\mu}-M_{1}\right)\left(i \gamma^{\mu} D_{\mu}-M_{2}\right)
$$

where

$$
M_{1}=\frac{-1+\sqrt{1+4 \xi \sigma}}{2 \xi}, \quad M_{2}=\frac{-1-\sqrt{1+4 \xi \sigma}}{2 \xi} .
$$

Here, we suppose that $\xi \geq-1 / 4 \sigma$. Then we can repre-

$$
G\left(x, x^{\prime}, \sigma\right)=(1+4 \xi \sigma)^{-1 / 2}\left\{S\left(x, x^{\prime}, M_{1}\right)-S\left(x, x^{\prime}, M_{2}\right)\right\},
$$


where the functions $S\left(x, x^{\prime}, M_{i}\right)$ obey the equation:

$$
\left(i \gamma^{\mu} D_{\mu}-M_{i}\right)_{x} S\left(x, x^{\prime}, M_{i}\right)=\delta\left(x-x^{\prime}\right)
$$

and $i=1,2$. This is exactly the usual GF of massive fermions in a constant external electromagnetic field, whose proper-time representation is well-known [5]. We have now derived all the preliminary formulae needed to construct the EP of our model.

\section{Dynamical symmetry break- ing without an external field}

In the absence of an electromagnetic field, the GF (12) in the proper-time representation has the form [5]:

$$
S\left(x-x^{\prime}, M_{i}\right)=-\int_{1 / \Lambda^{2}}^{\infty} \frac{d s}{(4 \pi s)^{2}} \exp \left[-i s M_{i}^{2}-\frac{i}{4 s}\left(x-x^{\prime}\right)^{2}\right]\left[M_{i}+\frac{1}{2 s} \gamma^{\mu}\left(x-x^{\prime}\right)_{\mu}\right]
$$

where $\Lambda$ is an ultraviolet cut-off parameter. It should be noted that, in contrast to the paper [18], where UV divergences have been dealt with by introducing a cut-off as a multiplier of the higher-derivative term, we have to insert in our present model this special parameter $\Lambda$ anyway. The point is that the action of our model contains only squares of derivatives and this is not enough in order to regularize one-loop Feynman graphs in a four-dimensional spacetime. Meanwhile the kinetic term of the model studied in [18] contains a cubic higher-derivative term which provides a complete regularization of any diagram.

The EP generated by the two functions $S\left(x, x^{\prime}, M_{i}\right)$ is the following

$$
V_{e f f}(\sigma)=-4 i(1+4 \xi \sigma)^{-1 / 2}\left[\int_{0}^{M_{1}} d m \operatorname{Sp} S(x, x, m)-\int_{0}^{M_{2}} d m \operatorname{Sp} S(x, x, m)\right],
$$

where the multiplier 4 is the dimension of the fermion representation, and the opposite sign of the second integral should be noted. After a Wick rotation, $i s \rightarrow s$, and integration over $m$, we get a positive expressions for both terms of EP (14) and no negative modes appear here. Therefore the EP can be written as

$$
V_{e f f}(\sigma)=\frac{\sigma^{2}}{2 \lambda}+\frac{1}{8 \pi^{2}} \int_{1 / \Lambda^{2}}^{\infty} \frac{d s}{s^{3}}\left(e^{-s M_{1}^{2}}+e^{-s M_{2}^{2}}\right) .
$$

Substituting here the expressions (10) and perfoming the integration over $s$, we finally obtain the effective potential (6)

$$
\begin{aligned}
& V_{e f f}(\sigma)= \frac{\sigma^{2}}{2 \lambda}+\frac{1}{16 \pi^{2} \xi^{4}}\left\{2 \Lambda^{4} \xi^{4}-2 \Lambda^{2} \xi^{2}(1+2 \xi \sigma)+\left(\frac{3}{2}-\gamma\right)\left[1+4 \xi \sigma+2(\xi \sigma)^{2}\right]\right. \\
&-\frac{1}{2}\left[1+4 \xi \sigma+2(\xi \sigma)^{2}-(1+2 \xi \sigma) \sqrt{1+4 \xi \sigma}\right] \ln \frac{1+2 \xi \sigma-\sqrt{1+4 \xi \sigma}}{2 \xi^{2} \Lambda^{2}} \\
&\left.\quad-\frac{1}{2}\left[1+4 \xi \sigma+2(\xi \sigma)^{2}+(1+2 \xi \sigma) \sqrt{1+4 \xi \sigma}\right] \ln \frac{1+2 \xi \sigma+\sqrt{1+4 \xi \sigma}}{2 \xi^{2} \Lambda^{2}}\right\},
\end{aligned}
$$


where we assume that $\sigma<<\Lambda$.

The gap equation

$$
V_{e f f}^{\prime}(\sigma)=0
$$

can be found directly and CS turns out to be broken for any value of the coupling constant and $\xi>0$, because

$$
V_{e f f}^{\prime}(0)=\frac{1}{4 \pi^{2} \xi^{3}}\left[-\Lambda^{2} \xi^{2}+1-\gamma+\ln \left(\Lambda^{2} \xi^{2}\right)\right]
$$

is always negative (see Fig. 1). This is an absolutely natural result because the higher-derivative term in the action (1) is noninvariant under chiral transformations, both of continuous and discrete type. It causes the presence of a bare current mass which is preserved even in the limit $\sigma \rightarrow 0$ when $M_{2}^{2} \rightarrow 1 / \xi^{2}$ being non-zero in all cases.

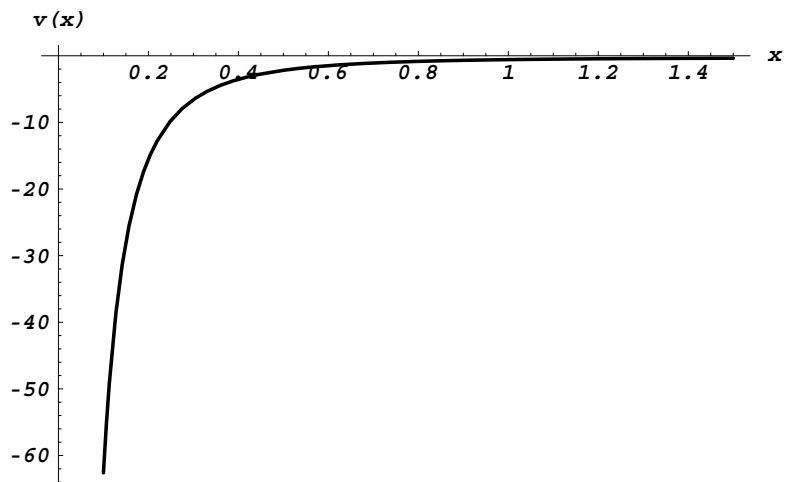

Figure 1. Plot of the derivative $v \equiv V_{e f f}^{\prime}(0) / \Lambda^{3}$ of the effective potential for $\sigma=0$ versus the dimensionless variable $x \equiv \Lambda^{2} \xi^{2}$. The fact that this derivative is always negative proves that chiral symmetry is always broken as well.

\section{Symmetry restoration un- der the influence of external constant magnetic or elec- tric fields}

The GF in an external constant magnetic field is given by $[5,10]$ :

$$
\begin{array}{r}
S\left(x-x^{\prime}, M_{i}\right)=-\int_{1 / \Lambda^{2}}^{\infty} \frac{d s}{(4 \pi s)^{2}} e^{-i s M_{i}^{2}} \exp \left(-\frac{i}{4 s}\left(x-x^{\prime}\right)_{\mu} C^{\mu \nu}\left(x-x^{\prime}\right)_{\nu}\right) \\
\times\left(M_{i}+\frac{1}{2 s} \gamma^{\mu} C_{\mu \nu}\left(x-x^{\prime}\right)^{\nu}-\frac{e}{2} \gamma^{\mu} F_{\mu \nu}\left(x-x^{\prime}\right)^{\nu}\right)\left(e B s \cot (e B s)-\frac{e s}{2} \gamma^{\mu} \gamma^{\nu} F_{\mu \nu}\right),
\end{array}
$$

where

$$
C_{\mu \nu}=\eta_{\mu \nu}+F_{\mu}^{\lambda} F_{\lambda \nu} \frac{1-e B s \cot (e B s)}{B^{2}} .
$$

Then, we can write the EP

$$
\begin{array}{r}
V_{e f f}(\sigma)=\frac{\sigma^{2}}{2 \lambda}+\frac{e B}{8 \pi^{2}} \int_{1 / \Lambda^{2}}^{\infty} \frac{d s}{s^{2}} \operatorname{coth}(e B s) \\
\times\left\{\exp \left[-s\left(\frac{\sqrt{1+4 \xi \sigma}+1}{2 \xi}\right)^{2}\right]+\exp \left[-s\left(\frac{\sqrt{1+4 \xi \sigma}-1}{2 \xi}\right)^{2}\right]\right\}
\end{array}
$$

The most reliable method to deal with the divergences is the cut-off scheme. We can make the following trick: to write the integral in the EP in the form

$$
\int_{1 / \Lambda^{2}}^{\infty} \frac{d s}{s^{3}} e^{-s M_{i}^{2}}\left[(e B s) \operatorname{coth}(e B s)-1-\frac{1}{3}(e B s)^{2}\right]+\int_{1 / \Lambda^{2}}^{\infty} \frac{d s}{s^{3}} e^{-s M_{i}^{2}}\left[1+\frac{1}{3}(e B s)^{2}\right]
$$


and to calculate the last one by keeping $\Lambda$ finite, while the first integral is finite already, so that we can set $1 / \Lambda^{2}=0$ at the lower limit. Then it appears to be possible to calculate it like a limit $\mu \rightarrow-1$, by using the formula

$$
\int_{0}^{\infty} d x x^{\mu-1} e^{-a x} \operatorname{coth}(c x)=\Gamma(\mu)\left[2^{1-\mu}(c)^{-\mu} \zeta\left(\mu, \frac{a}{2 c}\right)-a^{-\mu}\right] .
$$

After integration over $s$, we get

$$
\begin{aligned}
V_{e f f}(\sigma)= & \frac{\sigma^{2}}{2 \lambda}+\frac{1}{8 \pi^{2}}\left\{\Lambda^{4}+\frac{2}{3}(e B)^{2}\left[\ln \left(\frac{\Lambda^{2}}{2 e B}\right)-\gamma\right]\right. \\
& -\left(M_{1}^{2}+M_{2}^{2}\right) \Lambda^{2}+\frac{1}{2}\left(M_{1}^{4}+M_{2}^{4}\right)\left[\ln \left(\frac{\Lambda^{2}}{2 e B}\right)+1-\gamma\right] \\
& +e B\left[M_{1}^{2} \ln \left(\frac{M_{1}^{2}}{2 e B}\right)+M_{2}^{2} \ln \left(\frac{\left(M_{2}^{2}\right.}{2 e B}\right)\right] \\
& \left.-4(e B)^{2}\left[\zeta^{\prime}\left(-1, \frac{M_{1}^{2}}{2 e B}+1\right)+\zeta^{\prime}\left(-1, \frac{M_{2}^{2}}{2 e B}+1\right)\right]\right\}+\mathcal{O}\left(\frac{1}{\Lambda}\right),
\end{aligned}
$$

where

$$
\zeta^{\prime}(\nu, x)=\frac{d}{d \nu} \zeta(\nu, x)
$$

Substituting the expressions for $M_{1}^{2}$ and $M_{2}^{2}$, we obtain the following formula for the EP

$$
\begin{aligned}
V_{e f f}(\sigma)= & \frac{\sigma^{2}}{2 \lambda}+\frac{1}{8 \pi^{2}}\left\{\Lambda^{4}+\frac{2}{3}(e B)^{2}\left[\ln \left(\frac{\Lambda^{2}}{2 e B}\right)-\gamma\right]\right. \\
& -\frac{\Lambda^{2}}{\xi^{2}}(1+2 \xi \sigma)+\frac{1+4 \xi \sigma+2(\xi \sigma)^{2}}{2 \xi^{4}}\left[\ln \left(\frac{\Lambda^{2}}{2 e B}\right)+\frac{3}{2}-\gamma\right] \\
& +\frac{e B}{2 \xi^{2}}(1+2 \xi \sigma-\sqrt{1+4 \xi \sigma}) \ln \frac{1+2 \xi \sigma-\sqrt{1+4 \xi \sigma}}{4 \xi^{2} e B} \\
& +\frac{e B}{2 \xi^{2}}(1+2 \xi \sigma+\sqrt{1+4 \xi \sigma}) \ln \frac{1+2 \xi \sigma+\sqrt{1+4 \xi \sigma}}{4 \xi^{2} e B} \\
& -4(e B)^{2}\left[\zeta^{\prime}\left(-1,1+\frac{1+2 \xi \sigma-\sqrt{1+4 \xi \sigma}}{2 e B \xi^{2}}\right)\right. \\
& +\zeta^{\prime}\left(-1,1+\frac{1+2 \xi \sigma+\sqrt{1+4 \xi \sigma})]\}}{2 e B \xi^{2}}\right)
\end{aligned}
$$

To see if there are any possibilities for restoration of the chiral symmetry in this model, one should calculate the derivative $V_{e f f}^{\prime}(\sigma)$ at the origin $\sigma=0$ :

$$
\begin{array}{r}
V_{e f f}^{\prime}(0)=\frac{1}{8 \pi^{2} \xi^{3}}\left[2 \ln \left(\Lambda^{2} \xi^{2}\right)-2 \Lambda^{2} \xi^{2}+3-2 \gamma+2 e B \xi^{2}-2 \ln \left(2 e B \xi^{2}\right)\right. \\
\left.-2 e B \xi^{2} \ln \left(2 e B \xi^{2}\right)+2 e B \xi^{2} \ln (2 \pi)-4 e B \xi^{2} \ln \Gamma\left(1+\frac{1}{2 e B \xi^{2}}\right)\right]
\end{array}
$$

It should be noted that this formula does not reproduce Eq. (18) in the limit $B \rightarrow 0$, due to the circumstance that it was actually calculated as the zero-order term in the power expansion on the dimensionless parameter $\sigma^{2} / e B$. Thus, one has to keep $e B$ finite here.

As is clear from Fig. 2, there is a rather big area of values of the magnetic field strength and the cutoff parameter where the derivative $V_{e f f}^{\prime}(0)$ is positive.
That indicates the CS restoration on the quantum level. The corresponding numerical analysis proves in fact that this rerstoration occurs continuously with magnetic field strength growth. This means that there is a second-order phase transition as shown in Fig. 3. The same type of phase transition induced by a change of the nonlinearity parameter $\xi$ is depicted in Fig. 4 . 


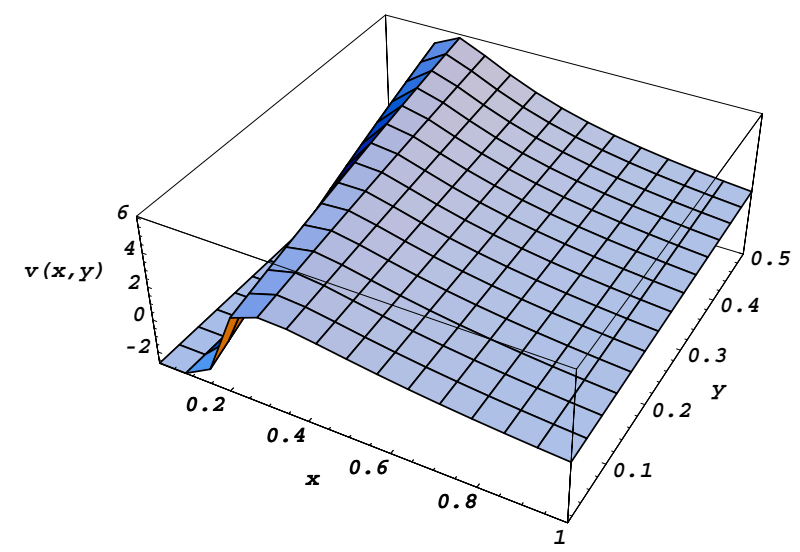

Figure 2. Plot of the derivative value $V_{e f f}^{\prime}(0) / \Lambda^{3}$ of the effective potential as a function of the two variables $x$ and $y \equiv e B \xi^{2}$. When the magnetic field is non-zero and for a reasonable rang of values of $x$, a domain is formed where the derivative of the potential is positive, signaling a phase transition accompanied by chiral symmetry restoration.

For the case of an external constant electric field, the EP has almost the same form:

$$
\begin{aligned}
V_{e f f}(\sigma)= & \frac{\sigma^{2}}{2 \lambda}+\frac{e E}{8 \pi^{2}} \int_{1 / \Lambda^{2}}^{\infty} \frac{d s}{s^{2}} \cot (e E s) \\
& \times\left\{\exp \left[-s\left(\frac{\sqrt{1+4 \xi \sigma}+1}{2 \xi}\right)^{2}\right]+\exp \left[-s\left(\frac{\sqrt{1+4 \xi \sigma}-1}{2 \xi}\right)^{2}\right]\right\}
\end{aligned}
$$

or, after substitution of the expressions for $M_{1}, M_{2}$,

$$
\begin{aligned}
V_{e f f}(\sigma)= & \frac{\sigma^{2}}{2 \lambda}+\frac{1}{8 \pi^{2}}\left\{\Lambda^{4}-\frac{2}{3}(e E)^{2}\left[\ln \left(\frac{\Lambda^{2}}{2 i e E}\right)-\gamma\right]\right. \\
& -\frac{\Lambda^{2}}{\xi^{2}}(1+2 \xi \sigma)+\frac{1+4 \xi \sigma+2(\xi \sigma)^{2}}{2 \xi^{4}}\left[\ln \left(\frac{\Lambda^{2}}{2 i e E}\right)+\frac{3}{2}-\gamma\right] \\
& +i \frac{e E}{2 \xi^{2}}(1+2 \xi \sigma-\sqrt{1+4 \xi \sigma}) \ln \frac{1+2 \xi \sigma-\sqrt{1+4 \xi \sigma}}{4 i \xi^{2} e E} \\
& +i \frac{e E}{2 \xi^{2}}(1+2 \xi \sigma+\sqrt{1+4 \xi \sigma}) \ln \frac{1+2 \xi \sigma+\sqrt{1+4 \xi \sigma}}{4 i \xi^{2} e E} \\
& +4(e E)^{2}\left[\zeta^{\prime}\left(-1,1-i \frac{1+2 \xi \sigma-\sqrt{1+4 \xi \sigma}}{2 e E \xi^{2}}\right)\right. \\
& \left.\left.+\zeta^{\prime}\left(-1,1-i \frac{1+2 \xi \sigma+\sqrt{1+4 \xi \sigma}}{2 e E \xi^{2}}\right)\right]\right\} .
\end{aligned}
$$

This expression has an imaginary part defining a particle creation velocity, and strictly speaking the vacuum becomes unstable [20]. However, for some small values of the electric field strength, when particle creation is still exponentially depressed, we can perform an anal- ysis of the DSB phenomenon using the real part of the EP.

To estimate if symmetry restoration takes place in an external electric field, we can use again the value of the derivative of the EP at the origin:

$$
\begin{aligned}
\operatorname{Re} V_{e f f}^{\prime}(0)= & \frac{1}{8 \pi^{2} \xi^{3}}\left[2 \ln \left(\Lambda^{2} \xi^{2}\right)-2 \Lambda^{2} \xi^{2}+3-2 \gamma\right. \\
& \left.-2 \ln \left(2 e E \xi^{2}\right)+\pi e E \xi^{2}+4 e E \xi^{2} \arg \Gamma\left(1-\frac{i}{2 e E \xi^{2}}\right)\right]
\end{aligned}
$$


Performing now a numerical analysis of the same fashion as in previous section, we may get qualitatively the same results. There are regions where CS is restored due to the electrical field effect. Being the procedure very similar, we do not present here explicit figures of that analysis for the sake of concissness.

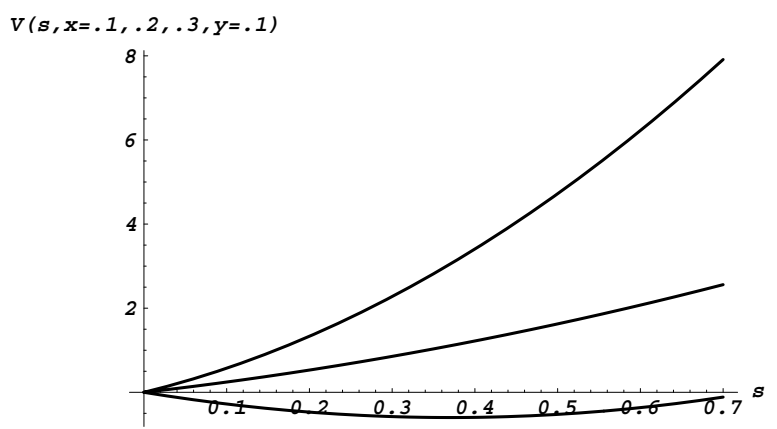

Figure 3. Plot of the effective potential $V(s) \equiv\left[V_{e f f}(\sigma)-\right.$ $\left.V_{e f f}(0)\right] / \Lambda^{4}$ as a function of $s \equiv \sigma / \Lambda$, for a particular value of $x=.15$ and three different values of $y=.05, .15, .3$, showing that the phase transition takes place.

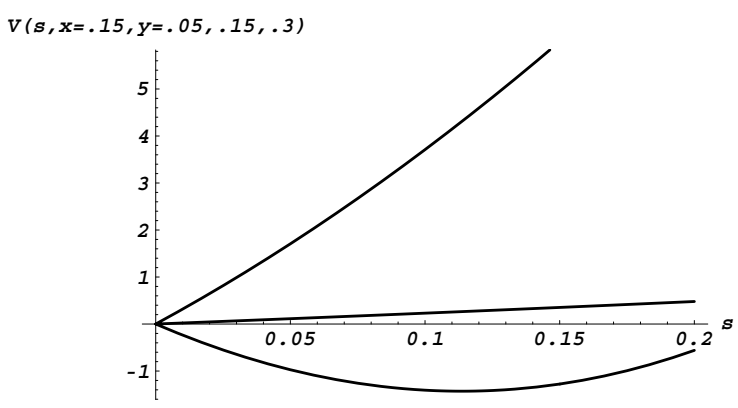

Figure 4. Plot of the potential $V(s)$ but now for a particular value of $y=.1$ and several values of $x=.1, .2, .3$.

\section{Conclusions}

We have studied in this paper the influence of magnetic and electric external fields on the CS restoration in a higher-derivative NJL-like model, where this symmetry is broken already in the absence of external fields. It has been shown that a domain of parameter values exists within the range of validity of our approximation where the external magnetic or electric fields restore chiral symmetry, at least at the quantum level. This is in contrast to the magnetic catalysis phenomenon occuring in the usual NJL model.
Fortunately the phase transition accompanying this symmetry restoration is a second order one and its continuous character reasures us on the correctness of our approximation. In fact, within the broken phase, for every value of $\Lambda$ there is some vicinity of the origin where $\sigma_{\min }<<\Lambda$, in accordance with the restrictions under which we have obtained the formula for the effective potential.

It should also be noted that it is not difficult to extend our model (and our calculational scheme) to include other higher derivative terms in the kinetic piece of the Lagrangian. In particular, one can consider a Lagrangian with a term of fourth order.

We would like to thank A.A. Andrianov for helpful discussions. This work has been partly financed by DGICYT (Spain), project PB96-0925, and by CIRIT (Generalitat de Catalunya), grant 1995SGR-00602. The work of Yu.I.Sh. was supported in part by Ministerio de Educación y Cultura (Spain), grant SB96-AN4620572. The work by S.D.O. has been partially supported by MEC (Spain). The work by S.P.G. has been partially supported by FAPESE (Brazil).

\section{References}

[1] Y. Nambu, G Jona-Lasinio, Phys. Rev. 122, 345 (1961).

[2] D. Gross, A. Neveu, Phys. Rev. D 10, 3235 (1974).

[3] E. Fahri and R. Jackiw, Eds., Dynamical Symmetry Breaking (World Scientific, Singapore, 1981); T. Muta and K. Yamawaki, Eds., Proceedings of the Workshop on Dynamical Symmetry Breaking (Nagoya, 1990); W. A. Bardeen, J. Kodaira and T. Muta, Eds., Proceedings of the International Workshop on Electroweak Symmetry Breaking (World Scientific, Singapore, 1991)

[4] M. Bando, T. Kugo, K. Yamawaki, Phys. Rep. 164, 217 (1988); B. Rosenstein, B. J. Warr, S. H. Park, Phys. Rep. 205, 59 (1991);T. Hatsuda, T. Kinuhiro, Phys. Rep. 247, 221 (1994); J. Bijnens, Phys. Rep. 265, 369 (1996).

[5] J. Schwinger, Phys. Rev. 82, 664 (1951).

[6] B.J. Harrington, S. Y. Park, A. Yildiz, Phys. Rev. D11, 1472 (1975); M. Stone, Phys. Rev. D 14, 3568 (1976); S. Kawati, G. Konisi, H. Miyata, Phys. Rev. D28 1537 (1983).

[7] S. P. Klevansky, R. H. Lemmer, Phys. Rev. D39, 3478 (1989); S. Klevansky, Rev. Mod. Phys. 64, 649 (1992).

[8] K. G. Klimenko, Theor. Math. Phys., 89, 211, 388 (1991);Z.Phys.C 54, 323 (1992); I. Krive and S. Naftulin, Phys. Rev. D46, 2337 (1992); K. Klimenko, A. S. Vshivtsev and B. V. Magnitsky, Nuovo Cim. A107, 439 (1994); JETP Lett. 61,871 (1995).

[9] H. Suganuma, T. Tatsumi, Ann. Phys. (NY) 208, 470 (1991);Progr. Theor. Phys. 90, 379 (1993). 
[10] D. Cangemi, G. Dunne, E. D'Hoker, Phys. Rev. D51, R2513 (1995); D52, R3163 (1995); V. P. Gusynin, V. A. Miransky, I.A. Shovkovoy, Nucl. Phys. B462, 249 (1996); C. N. Leung, Y. J. Ng and A. W. Ackly, Phys. Rev. D54, 4181 (1996);D.-S. Lee, C. N. Leung, Y. J. Ng, Phys. Rev. D55, 6504 (1997);

V. A. Miransky, hep-th/9805159.

[11] M. Ishi-i, T. Kashiwa and N. Tanemura, KYUSHUHET-40, hep-th/9707248; S. Kanemura, H.-T. Sato and H. Tochimura, Nucl.Phys. B517, 567 (1998).

[12] T. Muta, S. D. Odintsov, Mod. Phys. Lett. A6, 3641 (1991); C. T. Hill and D. S. Salopek, Ann. Phys. (NY), 213, 21 (1992); T. Inagaki, T. Muta, S. D. Odintsov, Mod. Phys. Lett. A8, 2117 (1993);E. Elizalde, S. D. Odintsov, Yu. I. Shil'nov, Mod. Phys. Lett. A9, 913 (1994); T. Inagaki, S. Mukaigawa and T. Muta, Phys. Rev. D52, R4267 (1996);E. Elizalde, S. Leseduarte, S. D. Odintsov, Yu. I. Shil'nov, Phys. Rev. D53, 1917 (1996); S. Kanemura and H.-T. Sato, Mod. Phys. Lett. A24,1777 (1995); G. Miele, P. Vitale, Nucl. Phys., B494, 365 (1997); M.A. Vdovichenko, A. S. Vshivtsev and K. G. Klimenko, JETP, 1998, to appear.

[13] I. L. Buchbinder, S. D. Odintsov, I. L. Shapiro, Effective Action in Quantum Gravity, (IOP Publishing, Bristol and Philadelphia, 1992.)

[14] T. Inagaki, T. Muta S. D. Odintsov, Dynamical Symmetry Breaking in Curved Spacetime, Prog. Theor. Phys. Suppl. 127, 93 (1997).
[15] D. M. Gitman, S. D. Odintsov, Yu. I. Shil'nov, Phys. Rev. D54, 2968 (1996); B. Geyer, L. N. Granda, S. D. Odintsov, Mod. Phys. Lett. A11, 2053 (1996); E. Elizalde, S. D. Odintsov, A. Romeo, Phys. Rev. D54, 4152 (1996);T. Inagaki, S. D. Odintsov, Yu. I. Shil'nov, KOBE-TH-97-02, hep-th/9709077;E. Elizalde, Yu. I. Shil'nov, V. V. Chitov, Class. Quant. Grav. 15, 735 (1998).

[16] M. Suzuki, Mod. Phys. Lett. A5, 1205 (1990); A. Hazenfrantz, K. Jansen, J. Kuti, Y. Shen, Nucl. Phys. B365, 79 (1991).

[17] A. A. Andrianov, V. A. Andrianov, Int. Journ. Mod. Phys. A8, 1981 (1993); A. A. Andrianov, V. A. Andrianov, V. L. Yudichev, Theor. Math. Phys. 108, 1069 (1996).

[18] T. Hamazaki, T. Kugo, Progr. Theor. Phys. 92, 645 (1994).

[19] E. Elizalde, S. Leseduarte, S. D. Odintsov, Phys. Lett. B347, 33 (1995).

[20] S.P. Gavrilov and D.M. Gitman, Phys. Rev. D53, 7162 (1996); E. S. Fradkin, D. M. Gitman and Sh. M. Schvartsman, Quantum Electrodynamics with unstable vacuum (Springer-Verlag, Berlin, 1991);S.P. Gavrilov, D.M. Gitman and A.E. Gonçalves, J. Math. Phys. 39, 3547 (1998). 